\title{
On Multivariate Grüss Inequalities
}

\author{
Chang-Jian Zhao' ${ }^{1}$ and Wing-Sum Cheung ${ }^{2}$ \\ ${ }^{1}$ Department of Information and Mathematics Sciences, College of Science, China Jiliang University, \\ Hangzhou 310018, China \\ ${ }^{2}$ Department of Mathematics, The University of Hong Kong, \\ Pokfulam Road, Hong Kong
}

Correspondence should be addressed to Chang-Jian Zhao, chjzhao@163.com

Received 6 March 2008; Revised 7 May 2008; Accepted 20 May 2008

Recommended by Martin Bohner

The main purpose of the present paper is to establish some new Grüss integral inequalities in $n$ independent variables. Our results in special cases yield some of the recent results on Pachpatte's, Mitrinović's, and Ostrowski's inequalities, and provide new estimates on such types of inequalities.

Copyright ( 12008 C.-J. Zhao and W.-S. Cheung. This is an open access article distributed under the Creative Commons Attribution License, which permits unrestricted use, distribution, and reproduction in any medium, provided the original work is properly cited.

\section{Introduction}

The well-known Grüss integral inequality [1] can be stated as follows (see [2, page 296]):

$$
\left|\frac{1}{b-a} \int_{a}^{b} f(x) g(x) d x-\left(\frac{1}{b-a} \int_{a}^{b} f(x) d x\right)\left(\frac{1}{b-a} \int_{a}^{b} g(x) d x\right)\right| \leq \frac{1}{4}(P-p)(Q-q),
$$

provided that $f$ and $g$ are two integrable functions on $[a, b]$ such that $p \leq f(x) \leq P, q \leq g(x) \leq$ $Q$, for all $x \in[a, b]$, where $p, P, q, Q$ are real constants.

Many generalizations, extensions, and variants of this inequality (1.1) have appeared in the literature, see [1-8] and the references given therein. The main purpose of the present paper is to establish several multivariate Grüss integral inequalities. Our results provide a new estimates on such type of inequalities.

\section{Main results}

In what follows, $\mathbb{R}$ denotes the set of real numbers, $\mathbb{R}^{n}$ the $n$-dimensional Euclidean space. Let $D=\left\{\left(x_{1}, \ldots, x_{n}\right): a_{i} \leq x_{i} \leq b_{i}(i=1, \ldots, n)\right\}$. For a function $u(x): \mathbb{R}^{n} \rightarrow \mathbb{R}$, we denote the 
first-order partial derivatives by $\left(\partial u(x) / \partial x_{i}\right)(i=1, \ldots, n)$ and $\int_{D} u(x) d x$ the $n$-fold integral $\int_{a_{1}}^{b_{1}} \cdots \int_{a_{n}}^{b_{n}} u\left(x_{1}, \ldots, x_{n}\right) d x_{1} \cdots d x_{n}$.

For continuous functions $p(x), q(x): D \rightarrow \mathbb{R}$ which are differentiable on $D$ and $w(x)$ : $D \rightarrow[0, \infty)$ an integrable function such that $\int_{D} w(x) d x>0$, we use the notation

$$
G[w, p, q]_{n}:=\int_{D} w(x) p(x) q(x) d x-\frac{\left(\int_{D} w(x) p(x) d x\right)\left(\int_{D} w(x) q(x) d x\right)}{\int_{D} w(x) d x}
$$

to simplify the details of presentation. Furthermore, if $\sum_{i=1}^{n}\left(\partial h / \partial x_{i}\right) \cdot\left(x_{i}-y_{i}\right) \neq 0$, for any $x, y \in$ $D$, we use the abbreviations

$$
\begin{aligned}
& G\left[\Sigma_{c}, w, g, h\right]_{n} \\
& \quad:=\frac{\int_{D}\left(\int_{D}\left(\sum_{i=1}^{n}\left(\partial f(c) / \partial x_{i}\right)\left(x_{i}-y_{i}\right) / \sum_{i=1}^{n}\left(\partial h(c) / \partial x_{i}\right)\left(x_{i}-y_{i}\right)\right) w(y) d y\right) g(x) h(x) w(x) d x}{\int_{D} w(y) d y} \\
& \quad-\frac{\int_{D}\left(\int_{D}\left(\sum_{i=1}^{n}\left(\partial f(c) / \partial x_{i}\right)\left(x_{i}-y_{i}\right) / \sum_{i=1}^{n}\left(\partial h(c) / \partial x_{i}\right)\left(x_{i}-y_{i}\right)\right) w(y) h(y) d y\right) g(x) w(x) d x}{\int_{D} w(y) d y}, \\
& G\left[\sum_{d}, w, f, h\right]_{n} \\
& \quad:=\frac{\int_{D}\left(\int_{D}\left(\sum_{i=1}^{n}\left(\partial g(d) / \partial x_{i}\right)\left(x_{i}-y_{i}\right) / \sum_{i=1}^{n}\left(\partial h(d) / \partial x_{i}\right)\left(x_{i}-y_{i}\right)\right) w(y) d y\right) f(x) h(x) w(x) d x}{\int_{D} w(y) d y} \\
& \quad-\frac{\int_{D}\left(\int_{D}\left(\sum_{i=1}^{n}\left(\partial g(d) / \partial x_{i}\right)\left(x_{i}-y_{i}\right) / \sum_{i=1}^{n}\left(\partial h(d) / \partial x_{i}\right)\left(x_{i}-y_{i}\right)\right) w(y) h(y) d y\right) f(x) w(x) d x}{\int_{D} w(y) d y} .
\end{aligned}
$$

It is clear that if

$$
\frac{\sum_{i=1}^{n}\left(\partial f(c) / \partial x_{i}\right)\left(x_{i}-y_{i}\right)}{\sum_{i=1}^{n}\left(\partial h(c) / \partial x_{i}\right)\left(x_{i}-y_{i}\right)}=\frac{\sum_{i=1}^{n}\left(\partial g(d) / \partial x_{i}\right)\left(x_{i}-y_{i}\right)}{\sum_{i=1}^{n}\left(\partial h(d) / \partial x_{i}\right)\left(x_{i}-y_{i}\right)}=1,
$$

then $G\left[\Sigma_{c}, w, g, h\right]_{n}=G[w, g, h]_{n}$ and $G\left[\Sigma_{d}, w, f, h\right]_{n}=G[w, f, h]_{n}$.

Our main results are established in the following theorems.

Theorem 2.1. Let $f, g, h: \mathbb{R}^{n} \rightarrow \mathbb{R}$ be continuous functions on $D$. If $f, g$ are differentiable on the interior of $D$ and $w(x): D \rightarrow[0, \infty)$ an integrable function such that $\int_{D} w(x) d x>0$. If $\sum_{i=1}^{n}\left(\partial h / \partial x_{i}\right) \cdot\left(x_{i}-y_{i}\right) \neq 0$, for every $x \in D$, then

$$
\left|G[w, f, g]_{n}\right| \leq \frac{1}{2}\left\{\left|G\left[\Sigma_{c}, w, g, h\right]_{n}\right|+\left|G\left[\Sigma_{d}, w, f, h\right]_{n}\right|\right\} .
$$

Proof. Let $x, y \in D$ with $x \neq y$. From the $n$-dimensional version of the Cauchy's mean value theorem (see [9]), we have

$$
\begin{aligned}
& f(x)-f(y)=\frac{\sum_{i=1}^{n}\left(\partial f(c) / \partial x_{i}\right)\left(x_{i}-y_{i}\right)}{\sum_{i=1}^{n}\left(\partial h(c) / \partial x_{i}\right)\left(x_{i}-y_{i}\right)}(h(x)-h(y)), \\
& g(x)-g(y)=\frac{\sum_{i=1}^{n}\left(\partial g(d) / \partial x_{i}\right)\left(x_{i}-y_{i}\right)}{\sum_{i=1}^{n}\left(\partial h(d) / \partial x_{i}\right)\left(x_{i}-y_{i}\right)}(h(x)-h(y)),
\end{aligned}
$$


where $c=\left(y_{1}+\alpha\left(x_{1}-y_{1}\right), \ldots, y_{n}+\alpha\left(x_{n}-y_{n}\right)\right)$ and $d=\left(y_{1}+\beta\left(x_{1}-y_{1}\right), \ldots, y_{n}+\beta\left(x_{n}-y_{n}\right)\right)(0<$ $\alpha<1,0<\beta<1$ ). Multiplying both sides of (2.5) by $g(x)$ and $f(x)$, respectively, and adding, we get

$$
\begin{aligned}
2 f(x) g(x)-g(x) f(y)-f(x) g(y)= & \frac{\sum_{i=1}^{n}\left(\partial f(c) / \partial x_{i}\right)\left(x_{i}-y_{i}\right)}{\sum_{i=1}^{n}\left(\partial h(c) / \partial x_{i}\right)\left(x_{i}-y_{i}\right)}(g(x) h(x)-g(x) h(y)) \\
& +\frac{\sum_{i=1}^{n}\left(\partial g(d) / \partial x_{i}\right)\left(x_{i}-y_{i}\right)}{\sum_{i=1}^{n}\left(\partial h(d) / \partial x_{i}\right)\left(x_{i}-y_{i}\right)}(f(x) h(x)-f(x) h(y)) .
\end{aligned}
$$

Multiplying both sides of (2.6) by $w(y)$ and integrating the resulting identity with respect to $y$ over $D$, we have

$$
\begin{aligned}
2\left(\int_{D} w(y) d y\right) f(x) g(x)-g(x) \int_{D} w(y) f(y) d y-f(x) \int_{D} w(y) g(y) d y \\
=\left(\int_{D} \frac{\sum_{i=1}^{n}\left(\partial f(c) / \partial x_{i}\right)\left(x_{i}-y_{i}\right)}{\sum_{i=1}^{n}\left(\partial h(c) / \partial x_{i}\right)\left(x_{i}-y_{i}\right)} w(y) d y\right) g(x) h(x) \\
\quad-g(x) \int_{D} \frac{\sum_{i=1}^{n}\left(\partial f(c) / \partial x_{i}\right)\left(x_{i}-y_{i}\right)}{\sum_{i=1}^{n}\left(\partial h(c) / \partial x_{i}\right)\left(x_{i}-y_{i}\right)} w(y) h(y) d y \\
\quad+\left(\int_{D} \frac{\sum_{i=1}^{n}\left(\partial g(d) / \partial x_{i}\right)\left(x_{i}-y_{i}\right)}{\sum_{i=1}^{n}\left(\partial h(d) / \partial x_{i}\right)\left(x_{i}-y_{i}\right)} w(y) d y\right) f(x) h(x) \\
\quad-f(x) \int_{D} \frac{\sum_{i=1}^{n}\left(\partial g(d) / \partial x_{i}\right)\left(x_{i}-y_{i}\right)}{\sum_{i=1}^{n}\left(\partial h(d) / \partial x_{i}\right)\left(x_{i}-y_{i}\right)} w(y) h(y) d y .
\end{aligned}
$$

Next, multiplying both sides of (2.7) by $w(x)$ and integrating the resulting identity with respect to $x$ over $D$, we have

$$
\begin{gathered}
2\left(\int_{D} w(y) d y\right) \int_{D} w(x) f(x) g(x) d x-\left(\int_{D} w(x) g(x) d x\right)\left(\int_{D} w(y) f(y) d y\right) \\
-\left(\int_{D} w(x) f(x) d x\right)\left(\int_{D} w(y) g(y) d y\right) \\
=\int_{D}\left(\int_{D} \frac{\sum_{i=1}^{n}\left(\partial f(c) / \partial x_{i}\right)\left(x_{i}-y_{i}\right)}{\sum_{i=1}^{n}\left(\partial h(c) / \partial x_{i}\right)\left(x_{i}-y_{i}\right)} w(y) d y\right) g(x) h(x) w(x) d x \\
-\int_{D}\left(\int_{D} \frac{\sum_{i=1}^{n}\left(\partial f(c) / \partial x_{i}\right)\left(x_{i}-y_{i}\right)}{\sum_{i=1}^{n}\left(\partial h(c) / \partial x_{i}\right)\left(x_{i}-y_{i}\right)} w(y) h(y) d y\right) g(x) w(x) d x \\
+\int_{D}\left(\int_{D} \frac{\sum_{i=1}^{n}\left(\partial g(d) / \partial x_{i}\right)\left(x_{i}-y_{i}\right)}{\sum_{i=1}^{n}\left(\partial h(d) / \partial x_{i}\right)\left(x_{i}-y_{i}\right)} w(y) d y\right) f(x) h(x) w(x) d x \\
-\int_{D}\left(\int_{D} \frac{\sum_{i=1}^{n}\left(\partial g(d) / \partial x_{i}\right)\left(x_{i}-y_{i}\right)}{\sum_{i=1}^{n}\left(\partial h(d) / \partial x_{i}\right)\left(x_{i}-y_{i}\right)} w(y) h(y) d y\right) f(x) w(x) d x .
\end{gathered}
$$


From (2.8), it is easy to observe that

$$
\left|G[w, f, g]_{n}\right| \leq \frac{1}{2}\left\{\left|G\left[\Sigma_{c}, w, g, h\right]_{n}\right|+\left|G\left[\Sigma_{d}, w, f, h\right]_{n}\right|\right\} .
$$

The proof is complete.

Remark 2.2. When $n=1$, we have $D=\left[a_{1}, b_{1}\right]$ and

$$
\frac{\sum_{i=1}^{n}\left(\partial f(c) / \partial x_{i}\right)\left(x_{i}-y_{i}\right)}{\sum_{i=1}^{n}\left(\partial h(c) / \partial x_{i}\right)\left(x_{i}-y_{i}\right)}=\frac{f^{\prime}(c)}{h^{\prime}(c)}, \quad \frac{\sum_{i=1}^{n}\left(\partial g(d) / \partial x_{i}\right)\left(x_{i}-y_{i}\right)}{\sum_{i=1}^{n}\left(\partial h(d) / \partial x_{i}\right)\left(x_{i}-y_{i}\right)}=\frac{g^{\prime}(d)}{h^{\prime}(d)}
$$

where $c=y_{1}+\alpha\left(x_{1}-y_{1}\right), 0<\alpha<1$, and $d=y_{1}+\beta\left(x_{1}-y_{1}\right), 0<\beta<1$. In this case, (2.4) reduces to the following inequality which was given by Pachpatte in [8]:

$$
|G[w, f, g]| \leq \frac{1}{2}\left\{\left\|\frac{f^{\prime}}{h^{\prime}}\right\|_{\infty}|G[w, g, h]|+\left\|\frac{g^{\prime}}{h^{\prime}}\right\|_{\infty}|G[w, f, h]|\right\},
$$

where $f(x), g(x), h(x):[a, b] \rightarrow \mathbb{R}$ are continuous on $[a, b]$ and differentiable in $(a, b), w:$ $[a, b] \rightarrow[0, \infty)$ is an integrable function with $\int_{a}^{b} w(x) d x>0,\|\cdot\|_{\infty}$ is the sup norm, and

$$
G[w, p, q]:=\int_{a}^{b} w(x) p(x) q(x) d x-\frac{\left(\int_{a}^{b} w(x) p(x) d x\right)\left(\int_{a}^{b} w(x) q(x) d x\right)}{\int_{a}^{b} w(x) d x} .
$$

Remark 2.3. If

$$
\frac{\sum_{i=1}^{n}\left(\partial f(c) / \partial x_{i}\right)\left(x_{i}-y_{i}\right)}{\sum_{i=1}^{n}\left(\partial h(c) / \partial x_{i}\right)\left(x_{i}-y_{i}\right)}=\frac{\sum_{i=1}^{n}\left(\partial g(d) / \partial x_{i}\right)\left(x_{i}-y_{i}\right)}{\sum_{i=1}^{n}\left(\partial h(d) / \partial x_{i}\right)\left(x_{i}-y_{i}\right)}=1
$$

we have $G\left[\Sigma_{c}, w, g, h\right]_{n}=G[w, f, g]_{n}$ and $G\left[\Sigma_{d}, w, f, h\right]_{n}=G[w, f, h]_{n}$. In this case, (2.4) reduces to the following interesting inequality:

$$
\left|G[w, f, g]_{n}\right| \leq \frac{1}{2}\left\{\left|G[w, g, h]_{n}\right|+\left|G[w, f, h]_{n}\right|\right\}
$$

Remark 2.4. If $h(x)=\sum_{i=1}^{n} x_{i}$, then (2.5) reduces to the following results, respectively,

$$
f(x)-f(y)=\sum_{i=1}^{n} \frac{\partial f(c)}{\partial x_{i}}\left(x_{i}-y_{i}\right), \quad g(x)-g(y)=\sum_{i=1}^{n} \frac{\partial g(d)}{\partial x_{i}}\left(x_{i}-y_{i}\right) .
$$

Furthermore, letting $w(y)=1,(2.7)$ reduces to

$$
\begin{array}{r}
\left|f(x) g(x)-\frac{1}{2 M} g(x) \int_{D} f(y) d y-\frac{1}{2 M} f(x) \int_{D} g(y) d y\right| \\
\leq \frac{1}{2 M} \sum_{i=1}^{n}\left(|g(x)|\left|\frac{\partial f}{\partial x_{i}}\left\|_{\infty}+|f(x)|\right\| \frac{\partial g}{\partial x_{i}} \|_{\infty}\right) E_{i}(x),\right.
\end{array}
$$


where $M=\operatorname{mes} D:=\prod_{i=1}^{n}\left(b_{i}-a_{i}\right)$, and $E_{i}(x):=\int_{D}\left|x_{i}-y_{i}\right| d y$. This is precisely a new inequality established by Pachpatte in [6]. If, in addition, $g(x) \equiv 1$, then inequality (2.16) reduces to the inequality established by Mitrinović in [2], which is in turn a generalization of the well-known Ostrowski inequality.

Theorem 2.5. Let $f, g, h$ be as in Theorem 2.1. Then,

$$
\begin{aligned}
& \left|G[w, f, g]_{n}\right| \leq \frac{1}{\left(\int_{D} w(y) d y\right)^{2}} \\
& \times \mid \int_{D}\left(w(x) h^{2}(x) \int_{D} \frac{\sum_{i=1}^{n}\left(\partial f(c) / \partial x_{i}\right)\left(x_{i}-y_{i}\right)}{\sum_{i=1}^{n}\left(\partial h(c) / \partial x_{i}\right)\left(x_{i}-y_{i}\right)} w(y) d y\right. \\
& \left.\cdot \int_{D} \frac{\sum_{i=1}^{n}\left(\partial g(d) / \partial x_{i}\right)\left(x_{i}-y_{i}\right)}{\sum_{i=1}^{n}\left(\partial h(d) / \partial x_{i}\right)\left(x_{i}-y_{i}\right)} w(y) d y\right) d x \\
& +\int_{D}\left(w(x) \int_{D} \frac{\sum_{i=1}^{n}\left(\partial f(c) / \partial x_{i}\right)\left(x_{i}-y_{i}\right)}{\sum_{i=1}^{n}\left(\partial h(c) / \partial x_{i}\right)\left(x_{i}-y_{i}\right)} w(y) h(y) d y\right. \\
& \left.\cdot \int_{D} \frac{\sum_{i=1}^{n}\left(\partial g(d) / \partial x_{i}\right)\left(x_{i}-y_{i}\right)}{\sum_{i=1}^{n}\left(\partial h(d) / \partial x_{i}\right)\left(x_{i}-y_{i}\right)} w(y) h(y) d y\right) d x \\
& -2 \int_{D}\left(w(x) h(x) \int_{D} \frac{\sum_{i=1}^{n}\left(\partial f(c) / \partial x_{i}\right)\left(x_{i}-y_{i}\right)}{\sum_{i=1}^{n}\left(\partial h(c) / \partial x_{i}\right)\left(x_{i}-y_{i}\right)} w(y) d y\right. \\
& \left.\cdot \int_{D} \frac{\sum_{i=1}^{n}\left(\partial g(d) / \partial x_{i}\right)\left(x_{i}-y_{i}\right)}{\sum_{i=1}^{n}\left(\partial h(d) / \partial x_{i}\right)\left(x_{i}-y_{i}\right)} w(y) h(y) d y\right) d x \mid .
\end{aligned}
$$

Proof. Multiplying both sides of (2.5) by $w(y)$ and integrate the resulting identities with respect to $y$ on $D$, we get, respectively,

$$
\begin{aligned}
& \left(\int_{D} w(y) d y\right) f(x)-\int_{D} w(y) f(y) d y \\
& \quad=h(x) \int_{D} \frac{\sum_{i=1}^{n}\left(\partial f(c) / \partial x_{i}\right)\left(x_{i}-y_{i}\right)}{\sum_{i=1}^{n}\left(\partial h(c) / \partial x_{i}\right)\left(x_{i}-y_{i}\right)} w(y) d y-\int_{D} \frac{\sum_{i=1}^{n}\left(\partial f(c) / \partial x_{i}\right)\left(x_{i}-y_{i}\right)}{\sum_{i=1}^{n}\left(\partial h(c) / \partial x_{i}\right)\left(x_{i}-y_{i}\right)} w(y) h(y) d y \\
& \left(\int_{D} w(y) d y\right) g(x)-\int_{D} w(y) g(y) d y \\
& \quad=h(x) \int_{D} \frac{\sum_{i=1}^{n}\left(\partial g(d) / \partial x_{i}\right)\left(x_{i}-y_{i}\right)}{\sum_{i=1}^{n}\left(\partial h(d) / \partial x_{i}\right)\left(x_{i}-y_{i}\right)} w(y) d y-\int_{D} \frac{\sum_{i=1}^{n}\left(\partial g(d) / \partial x_{i}\right)\left(x_{i}-y_{i}\right)}{\sum_{i=1}^{n}\left(\partial h(d) / \partial x_{i}\right)\left(x_{i}-y_{i}\right)} w(y) h(y) d y
\end{aligned}
$$


Multiplying the left sides and right sides of (2.18), we get

$$
\begin{aligned}
&\left(\int_{D} w(y) d y\right)^{2} f(x) g(x)-\left(\int_{D} w(y) d y\right) f(x)\left(\int_{D} w(y) g(y) d y\right) \\
&-\left(\int_{D} w(y) d y\right) g(x)\left(\int_{D} w(y) f(y) d y\right)+\left(\int_{D} w(y) f(y) d y\right)\left(\int_{D} w(y) g(y) d y\right) \\
&= h^{2}(x) \int_{D} \frac{\sum_{i=1}^{n}\left(\partial f(c) / \partial x_{i}\right)\left(x_{i}-y_{i}\right)}{\sum_{i=1}^{n}\left(\partial h(c) / \partial x_{i}\right)\left(x_{i}-y_{i}\right)} w(y) d y \cdot \int_{D} \frac{\sum_{i=1}^{n}\left(\partial g(d) / \partial x_{i}\right)\left(x_{i}-y_{i}\right)}{\sum_{i=1}^{n}\left(\partial h(d) / \partial x_{i}\right)\left(x_{i}-y_{i}\right)} w(y) d y \\
&+\int_{D} \frac{\sum_{i=1}^{n}\left(\partial f(c) / \partial x_{i}\right)\left(x_{i}-y_{i}\right)}{\sum_{i=1}^{n}\left(\partial h(c) / \partial x_{i}\right)\left(x_{i}-y_{i}\right)} w(y) h(y) d y \cdot \int_{D} \frac{\sum_{i=1}^{n}\left(\partial g(d) / \partial x_{i}\right)\left(x_{i}-y_{i}\right)}{\sum_{i=1}^{n}\left(\partial h(d) / \partial x_{i}\right)\left(x_{i}-y_{i}\right)} w(y) h(y) d y \\
&-h(x) \int_{D} \frac{\sum_{i=1}^{n}\left(\partial g(d) / \partial x_{i}\right)\left(x_{i}-y_{i}\right)}{\sum_{i=1}^{n}\left(\partial h(d) / \partial x_{i}\right)\left(x_{i}-y_{i}\right)} w(y) d y \cdot \int_{D} \frac{\sum_{i=1}^{n}\left(\partial f(c) / \partial x_{i}\right)\left(x_{i}-y_{i}\right)}{\sum_{i=1}^{n}\left(\partial h(c) / \partial x_{i}\right)\left(x_{i}-y_{i}\right)} w(y) h(y) d y \\
&-h(x) \int_{D} \frac{\sum_{i=1}^{n}\left(\partial f(c) / \partial x_{i}\right)\left(x_{i}-y_{i}\right)}{\sum_{i=1}^{n}\left(\partial h(c) / \partial x_{i}\right)\left(x_{i}-y_{i}\right)} w(y) d y \cdot \int_{D} \frac{\sum_{i=1}^{n}\left(\partial g(d) / \partial x_{i}\right)\left(x_{i}-y_{i}\right)}{\sum_{i=1}^{n}\left(\partial h(d) / \partial x_{i}\right)\left(x_{i}-y_{i}\right)} w(y) h(y) d y .
\end{aligned}
$$

Multiplying both sides of (2.19) by $w(x)$ and integrating the resulting identity with respect to $x$ over $D$, we get

$$
\begin{aligned}
& \left(\int_{D} w(y) d y\right)^{2} \int_{D} w(x) f(x) g(x) d x-\left(\int_{D} w(y) d y\right)\left(\int_{D} w(x) f(x) d x\right)\left(\int_{D} w(y) g(y) d y\right) \\
& -\left(\int_{D} w(y) d y\right)\left(\int_{D} w(x) g(x) d x\right)\left(\int_{D} w(y) f(y) d y\right) \\
& +\left(\int_{D} w(x) d x\right)\left(\int_{D} w(y) f(y) d y\right)\left(\int_{D} w(y) g(y) d y\right) \\
& =\int_{D}\left(w(x) h^{2}(x) \int_{D} \frac{\sum_{i=1}^{n}\left(\partial f(c) / \partial x_{i}\right)\left(x_{i}-y_{i}\right)}{\sum_{i=1}^{n}\left(\partial h(c) / \partial x_{i}\right)\left(x_{i}-y_{i}\right)} w(y) d y \cdot \int_{D} \frac{\sum_{i=1}^{n}\left(\partial g(d) / \partial x_{i}\right)\left(x_{i}-y_{i}\right)}{\sum_{i=1}^{n}\left(\partial h(d) / \partial x_{i}\right)\left(x_{i}-y_{i}\right)} w(y) d y\right) d x \\
& +\int_{D}\left(w(x) \int_{D} \frac{\sum_{i=1}^{n}\left(\partial f(c) / \partial x_{i}\right)\left(x_{i}-y_{i}\right)}{\sum_{i=1}^{n}\left(\partial h(c) / \partial x_{i}\right)\left(x_{i}-y_{i}\right)} w(y) d y \cdot \int_{D} \frac{\sum_{i=1}^{n}\left(\partial g(d) / \partial x_{i}\right)\left(x_{i}-y_{i}\right)}{\sum_{i=1}^{n}\left(\partial h(d) / \partial x_{i}\right)\left(x_{i}-y_{i}\right)} w(y) h(y) d y\right) d x \\
& -\int_{D}\left(w(x) h(x) \int_{D} \frac{\sum_{i=1}^{n}\left(\partial g(d) / \partial x_{i}\right)\left(x_{i}-y_{i}\right)}{\sum_{i=1}^{n}\left(\partial h(d) / \partial x_{i}\right)\left(x_{i}-y_{i}\right)} w(y) d y \cdot \int_{D} \frac{\sum_{i=1}^{n}\left(\partial f(c) / \partial x_{i}\right)\left(x_{i}-y_{i}\right)}{\sum_{i=1}^{n}\left(\partial h(c) / \partial x_{i}\right)\left(x_{i}-y_{i}\right)} w(y) h(y) d y\right) d x \\
& -\int_{D}\left(w(x) h(x) \int_{D} \frac{\sum_{i=1}^{n}\left(\partial f(c) / \partial x_{i}\right)\left(x_{i}-y_{i}\right)}{\sum_{i=1}^{n}\left(\partial h(c) / \partial x_{i}\right)\left(x_{i}-y_{i}\right)} w(y) d y \cdot \int_{D} \frac{\sum_{i=1}^{n}\left(\partial g(d) / \partial x_{i}\right)\left(x_{i}-y_{i}\right)}{\sum_{i=1}^{n}\left(\partial h(d) / \partial x_{i}\right)\left(x_{i}-y_{i}\right)} w(y) h(y) d y\right) d x .
\end{aligned}
$$

From (2.20), it is easy to arrive at inequality (2.17). The proof of Theorem 2.5 is completed. 
Remark 2.6. Taking $n=1$, we have $D=\left[a_{1}, b_{1}\right]$ and

$$
\frac{\sum_{i=1}^{n}\left(\partial f(c) / \partial x_{i}\right)\left(x_{i}-y_{i}\right)}{\sum_{i=1}^{n}\left(\partial h(c) / \partial x_{i}\right)\left(x_{i}-y_{i}\right)}=\frac{f^{\prime}(c)}{h^{\prime}(c)}, \quad \frac{\sum_{i=1}^{n}\left(\partial g(d) / \partial x_{i}\right)\left(x_{i}-y_{i}\right)}{\sum_{i=1}^{n}\left(\partial h(d) / \partial x_{i}\right)\left(x_{i}-y_{i}\right)}=\frac{g^{\prime}(d)}{h^{\prime}(d)},
$$

where $c=y_{1}+\alpha\left(x_{1}-y_{1}\right), 0<\alpha<1$, and $d=y_{1}+\beta\left(x_{1}-y_{1}\right), 0<\beta<1$. In this case, (2.20) becomes the following inequality which was given by Pachpatte in [8]:

$$
|G[w, f, g]| \leq\left|\int_{a}^{b} w(x) h^{2}(x) d x-\frac{\left(\int_{a}^{b} w(x) h(x) d x\right)^{2}}{\int_{a}^{b} w(x) d x}\right|\left\|\frac{f^{\prime}}{g^{\prime}}\right\|_{\infty}\left\|\frac{g^{\prime}}{h^{\prime}}\right\|_{\infty}
$$

where $f(x), g(x), h(x):[a, b] \rightarrow \mathbb{R}$ are continuous on $[a, b]$ and differentiable in $(a, b), w:$ $[a, b] \rightarrow[0, \infty)$ is an integrable function with $\int_{a}^{b} w(x) d x>0$, and

$$
G[w, p, q]:=\int_{a}^{b} w(x) p(x) q(x) d x-\frac{\left(\int_{a}^{b} w(x) p(x) d x\right)\left(\int_{a}^{b} w(x) q(x) d x\right)}{\int_{a}^{b} w(x) d x} .
$$

Remark 2.7. If $h(x)=\sum_{i=1}^{n} x_{i}$, then (2.5) becomes

$$
f(x)-f(y)=\sum_{i=1}^{n} \frac{\partial f(c)}{\partial x_{i}}\left(x_{i}-y_{i}\right), \quad g(x)-g(y)=\sum_{i=1}^{n} \frac{\partial g(d)}{\partial x_{i}}\left(x_{i}-y_{i}\right) .
$$

Multiplying the left and right sides of (2.24), we get

$$
f(x) g(x)-f(x) g(y)-g(x) f(y)+f(y) g(y)=\left[\sum_{i=1}^{n} \frac{\partial f(c)}{\partial x_{i}}\left(x_{i}-y_{i}\right)\right]\left[\sum_{i=1}^{n} \frac{\partial g(d)}{\partial x_{i}}\left(x_{i}-y_{i}\right)\right]
$$

Integrating both sides of (2.25) with respect to $y$ on $D$, we have the following inequality which was established by Pachpatte in [6]:

$$
\begin{gathered}
\left|f(x) g(x)-f(x)\left(\frac{1}{M} \int_{D} g(y) d y\right)-g(x)\left(\frac{1}{M} \int_{D} f(y) d y\right)+\frac{1}{M} \int_{D} f(y) g(y) d y\right| \\
\leq \frac{1}{M} \int_{D}\left\{\left[\sum_{i=1}^{n}\left\|\frac{\partial f}{\partial x_{i}}\right\|_{\infty}\left|x_{i}-y_{i}\right|\right]\left[\sum_{i=1}^{n}\left\|\frac{\partial g}{\partial x_{i}}\right\|_{\infty}\left|x_{i}-y_{i}\right|\right]\right\} d y,
\end{gathered}
$$

where $M=\operatorname{mes} D=\prod_{i=1}^{n}\left(b_{i}-a_{i}\right)$.

\section{Acknowledgments}

The authors cordially thank the anonymous referee for his/her valuable comments which led to the improvement of this paper. Research is supported by Zhejiang Provincial Natural Science Foundation of China (Y605065), Foundation of the Education Department of Zhejiang Province of China (20050392). Research is partially supported by the Research Grants Council of the Hong Kong SAR, China (Project no. HKU7016/07P). 


\section{References}

[1] G. Grüss, "Über das Maximum des absoluten Betrages von $1 /(b-a) \int_{a}^{b} f(x) g(x) d x-1 /(b-a)^{2}$ $\times \int_{a}^{b} f(x) d x \int_{a}^{b} g(x) d x, "$ Mathematische Zeitschrift, vol. 39, no. 1, pp. 215-226, 1935.

[2] D. S. Mitrinović, J. E. Pečarić, and A. M. Fink, Classical and New Inequalities in Analysis, vol. 61 of Mathematics and Its Applications, Kluwer Acadmic Punlishers, Dordrecht, The Netherlands, 1993.

[3] S. S. Dragomir, "Some integral inequalities of Grüss type," Indian Journal of Pure and Applied Mathematics, vol. 31, no. 4, pp. 397-415, 2000.

[4] A. M. Fink, "A treatise on Grüss' inequality," in Analytic and Geometric Inequalities and Applications, T. M. Rassias and H. M. Srivastava, Eds., vol. 478 of Mathematics and Its Applications, pp. 93-113, Kluwer Acadmic Punlishers, Dordrecht, The Netherlands, 1999.

[5] B. G. Pachpatte, "On Grüss type inequalities for double integrals," Journal of Mathematical Analysis and Applications, vol. 267, no. 2, pp. 454-459, 2002.

[6] B. G. Pachpatte, "On multivariate Ostrowski type inequalities," Journal of Inequalities in Pure and Applied Mathematics, vol. 3, no. 4, article 58, 5 pages, 2002.

[7] B. G. Pachpatte, "New weighted multivariate Grüss type inequalities," Journal of Inequalities in Pure and Applied Mathematics, vol. 4, no. 5, article 108, 9 pages, 2003.

[8] B. G. Pachpatte, "A note on Grüss type inequalities via Cauchy's mean value theorem," Mathematical Inequalities E Applications, vol. 11, no. 1, pp. 75-80, 2008.

[9] W. Rudin, Principles of Mathematical Analysis, International Series in Pure and Applied Mathematics, McGraw-Hill, New York, NY, USA, 1953. 